\title{
Comparación de microbio-algoritmos para redes ópticas de transporte de datos
}

\author{
Arturo B. Rodríguez ${ }^{1 *}$, Leonardo J. Ramírez ${ }^{2}$ y Gabriel A. Puerta ${ }^{2}$ \\ (1) Facultad Tecnológica, Dpto. de Tecnologías Industriales, Universidad de Santiago de Chile, Av. Ecuador №3369, \\ Santiago-Chile. (correo-e: arturo.rodriguez@usach.cl) \\ (2) Facultad de Ingeniería, Universidad Militar de Nueva Granada, Colombia. \\ (correo-e: leonardo.ramirez@unimilitar.edu.co; u7700114@unimilitar.edu.co)
}

Recibido Dic. 30, 2019; Aceptado Mar. 2, 2020; Versión final Mar. 20, 2020, Publicado Jun. 2020

\section{Resumen}

El objetivo del presente trabajo es comparar las heurísticas del tipo microbioheurísticas utilizando un escenario con trafico dinámico en la red National Science Foundation NETwork (NSFNET). Se utilizaron diversos indicadores tales como: probabilidad de bloqueo promedio, utilización de la red promedio y la tasa de uso de la red óptica. Se compararon los algoritmos genéticos, flagelos bacteriales y de hifas fúngicas. Los resultados muestran al algoritmo genético con mejor respuesta al bloqueo y el algoritmo basado en hifas fúngicas con mejor uso de los recursos de la red. El algoritmo basado en flagelos bacteriales muestra un desempeño sin diferencias claras, excepto en el intervalo de 80 a 130 erlangs donde la probabilidad de bloqueo es bastante menor. En conclusión, estos algoritmos pueden ser utilizados en otros procesos del ámbito industrial.

\section{Comparison of microbio-algorithms for data transport optical networks}

\begin{abstract}
The objective of the present study is to compare heuristics of microbioheuristic type employing a scenario with dynamic traffic in the National Science Foundation NETwork (NSFNET) network. Several indicators are used such as the average blocking probability, the usage of the red average, and the usage rate of the red optic. Genetic algorithms, bacterial flagella, and fungal hyphae were compared. The results show that the genetic algorithm has the best response to blocking and that the algorithm based on fungal hyphae has the best use of network resources. The algorithm based on bacterial flagella shows a performance without clear differences, except in the range of 80 to 130 erlangs, where the probability of blocking is much lower. In conclusion, these algorithms can be used for other industrial processes.
\end{abstract}




\section{INTRODUCCIÓN}

El centro de la discusión del transporte de datos en redes ópticas es la conmutación de paquetes; la cual es preferible dada su alta velocidad de respuesta frente al routing. Cada vez se las capas superiores se acercan a la capa física utilizando cada vez menos elementos que ralentizan el procesamiento de paquetes en la transmisión o recepción, es por eso que la capa enlace cobra cada día mayor importancia. Una de las consecuencias de esta evolución es la desaparición de la capa IP, en vista que cada vez el routing es menos necesario, sin embargo, la dirección IP deberá permanecer para solventar el necesario etiquetamiento de los dispositivos para garantizar un correcto transporte de origen a destino(Xiaoweng et al., 2003). El transporte de datos se ha evolucionado del dominio electrónico (DOMEL) al dominio óptico (DOMOP), esta evolución determino y marco diferencias en las tecnologías de transporte, mientras el routing está asociado al DOMEL el switching se asocia al DOMOP. Los algoritmos de enrutamiento para estas tecnologías se diferencian debido a las restricciones que cada dominio entrega.

Los algoritmos convencionales como dijkstra, Floy Marshall entre otros desarrollan sus procesos computacionales buscando la optimización de una función aptitud basada en algún criterio tal como, retardo, saltos, etc., las desventajas de estos procesos es que solo encuentran una solución, esto revela una debilidad puesto que si la ruta seleccionada estuviera bajo falla los nodos extremos deberán resolver el problema recalculando el algoritmo, además requieren de un universo de soluciones estático debido a su búsqueda del óptimo. Sin embargo, las redes de transporte óptico tienen restricciones propias de la tecnología como no tener buffers, es decir cada solicitud que llega a un nodo tiene que ser atendida, de lo contrario debe ser bloqueada y el dispositivo solicitante deberá volver a solicitar conexión (Zhou et al., 2018). Adicionalmente el tráfico en las redes ópticas es de características dinámicas, es decir que constantemente se ocupan y desocupan los enlaces en diferentes longitudes de onda, dando un enorme dinamismo al universo de soluciones, no permitiendo su optimización. Esto generó la aparición de los algoritmos heurísticos muy utilizando en la industria en la resolución de los problemas de enrutamiento de las redes de transporte, las ventajas que tienen frente a los convencionales es la gran cantidad de soluciones en un mismo proceso algorítmico y la rapidez de la solución, sin embargo, la gran desventaja es que estos algoritmos no encuentran óptimos absolutos algunas veces solo locales e inclusive solo buenas soluciones. Las redes de transporte óptico deben atender las solicitudes lo antes posible, es por eso que las heurísticas responden correctamente a la demanda óptica.

De esta perspectiva se han utilizado con mucha intensidad diferentes algoritmos, entre ellos podemos ver Simulated Annealing, Búsqueda Tabu, Ant system, Bat, Firefly, Snake One, Snake Two, Snake Three y otros por lo general basados en la búsqueda del menor costo o retardo en la ruta. Desde la perspectiva del tráfico que soporta una red, puede ser clasificada como de tráfico estático (static traffic-ST) o dinámico (dynamic traffic-DT), las ST tienen el universo de soluciones poco variante en el tiempo, mientras las DT tienen un universo de soluciones dinámico. Por eso las redes de transporte actuales utilizan algoritmos de enrutamiento heurísticos. Para la comparación efectiva de estos algoritmos se utiliza la probabilidad de bloqueo $(\mathrm{PdB})$ y la utilización de la red $(\mathrm{UdR})$. El problema del enrutamiento se denomina RWA (Routing Wavelength Assignement), su importancia radica en a la necesidad de seleccionar una ruta y una longitud de onda asociada para el establecimiento de la conexión.

En las redes totalmente ópticas (AON- All Optical Network) los transmitter o transmisores están conectados con cada receptor en todas las longitudes de onda y por lo tanto se requieren tantas señales como longitudes de onda existen. Cuando muchas sesiones utilizan la misma longitud de onda estas señales no se pueden enviar sobre la misma fibra simultáneamente para evitar las colisiones dentro de la red y por consecuencia el bloqueo de la sesión. Se debe asegurar que, si un receptor está en espera de data en una la longitud de onda determinada en un intervalo de tiempo determinado, solo una señal con esa longitud de onda debe llegar al receptor, si 2 o más señales llegan bajo esas condiciones estamos ante un fenómeno llamado contencion. Esta se puede evitar aislando las señales espaciales y/o temporalmente. Es decir, enviando la señal por otro camino y/o en otro tiempo, el asilamiento temporal por lo general es lo que se quiere evitar pues trae consigo latencia (Brigitte et al., 2017; Piedrahita et al., 2014; Russo et al., 2011).

Bajo esta condición se establecen dos restricciones, la primera CCW (Continuity Constraint Wavelength) que determina el uso de la misma longitud de onda en cada enlace componente de la ruta y la segunda RUW (Reuse of Wavelength) donde es permitido diferentes longitudes de onda a lo largo de toda la ruta. Pero, se genera un aumento en la utilización de la red aumentando la probabilidad de bloqueo de la demanda futura; ya que los caminos se agotan mientras más rutas se establezcan. El presente trabajo estudia y propone una nueva clasificación de heurísticas, que utilizan los elementos microbiológicos como medio para el desarrollo de algoritmos como son los genéticos (GEA) (Simon et al., 2010), flagelos bacterianos (FBA) y algoritmos basados en las hifas de los hongos (HYA). Los resultados son interesantes y proponen una nueva taxonomía que permitirá el desarrollo de una nueva línea de heurísticas (Rodriguez et al., 2019). 


\section{EL PROBLEMA RWA}

La red de transporte óptico está formada por switches denominados OXC (Optical Cross Connect) o conmutadores ópticos, enlazados por fibras ópticas. Estos sistemas utilizan la misma longitud de onda en cada enlace, pero se han desarrollado sistemas de enrutamiento con reutilización de la longitud de onda, donde se permite cambiarla, sin embargo, los estudios no muestran cambios significativos en el rendimiento del enrutamiento (Balasis et al., 013).

\section{Descripción del problema}

En la Figura 1, se observan 6 OXC, que forman parte de un sistema mayor, las rutas establecidas con su longitud de onda respectiva (lightpath) son (E-D-A-B-C/ $\left.\lambda_{1}\right)$; (D-A-B-F/ $\left.\lambda_{0}\right)$ y $\left(D-A-B-C / \lambda_{2}\right)$; llega una solicitud de servicio cuya solución pasa por los enlaces (E-D-B-C),sin embargo por simple inspección se observa que no es posible encontrar una longitud de onda similar en los 3 enlaces, este fenómeno es denominado contención, por lo que la solución pasará por cambiar los enlaces o en diferentes tiempos. Por lo tanto, el problema RWA requiere encontrar una ruta y su longitud de onda asociada en un escenario de tráfico dinámico para redes sin conversión de longitud de onda, es decir con la restricción CCW (Assis et al., 2010).

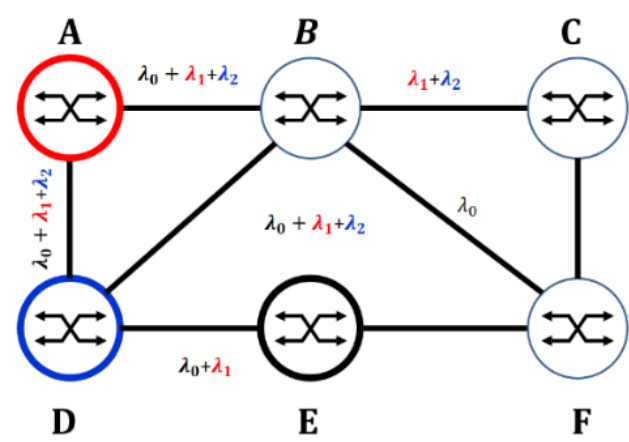

Fig. 1: Problema RWA

\section{Enrutamiento lumínico}

La solución del problema RWA está asociado a dos tipos de tráfico, para el ST la solución se denomina Static Lightpath Establishment (SLE) y para DT se denomina Dynamic Lighpath Establishment (DLE). En la litertura se aborda el problema desde la estrategia computacional, la heurística y/o algoritmo y el criterio de optimización. Respecto de la estrategia computacional se observan dos tipos la solución dividida y la integral, mientras la solución dividida desarrolla dos algoritmos para encontrar el lightpath, uno para encontrar la ruta y el otro para encontrar la longitud de onda asociada y la solución integral encuentra la ruta y la longitud de onda en un solo proceso algorítmico. Respecto de los algoritmos se pueden clasificar en dos grandes grupos, los convencionales que optimizan para el caso SLE y los heurísticos que solo encuentran una buena solución para el caso DLE, respecto de los criterios de optimización, se pueden encontrar por minimización de la probabilidad de bloqueo de nuevas solicitudes, minimización de la utilización de las longitud de onda de la red, minimización del uso de longitudes de onda por enlace minimización de la dispersión en la ruta y minimización del ruido ASE (Amplified Spontaneous Emission) entre otros (Duhamel et al., 2016; Koganti et al., 2014; Montes-Castañeda et al., 2015). Los algoritmos de enrutamiento se encuentran clasificados en convencionales y heurísticos, los convencionales tienen la ventaja de encontrar los óptimos absolutos de un universo de soluciones, pero la desventaja es que dicho universo debe estar estático en caso contrario se imposibilita la optimización, otra desventaja es que solo encuentran una solución y en caso de una caída de un enlace seleccionado el recalculo de la ruta a través del algoritmo se hace necesario.

\section{Demanda de la red}

La demanda de una red es aquel conjunto de solicitudes que llegan a los nodos fronterizos (Edge Node), estas traen consigo tres parámetros, el nodo destino, la cantidad de canales solicitados y el tiempo de conexión solicitado, esto es definido en la ecuación 1 a continuación.

$d_{i}^{r o}=\left(r_{D}, n_{C}, t_{C}\right)$

Dónde: $d_{i}{ }^{r}$ es el vector que representa la i-ésima solicitud que llega al nodo $r_{0}$ fronterizo (Edge Router), $r_{D}$ es el número de identificación del nodo destino de la solicitud de llegada, $n_{C}$ es el número de conexiones solicitada para el par $\left(r_{o}, r_{D}\right)$ y $n_{C}$ es el tiempo de conexión solicitada. Para el desarrollo del modelamiento y 
simulación se establecieron cuatro matrices, la matriz de enlaces $E$ que determina la topología de la red a utilizar, la Matriz de costos $C$ que monitorea el estado de los costos de los enlaces estará siempre estableciendo la capacidad disponible de los enlaces, la matriz de longitud de onda $\lambda$; que determinará la longitud de onda en uso y la matriz de tiempos $T$ que monitorea los tiempos restantes de conexión, estas matrices son actualizadas dinámicamente, la estrucutra de la matriz de tiempos es análoga a la matriz $\lambda$, pero sus elementos serán de valor 1 cuando se encuentren disponibles las longitudes de onda, en el enlace dado, y el tiempo será negativo en decremento cuando se encuentre en uso. A continuación se muestran las definiciones de las matrices.

$E=\left\{\begin{array}{cc}e_{i j} / e_{i j} & e_{i j}=G N E E \wedge e_{i j}=1 \\ \forall i \in[0, N-1] & \wedge j \in[0, N-1]\end{array}\right\}$

Donde : $N$ es el número de nodos de la red, $G$ es número muy grande, NEE es No Existe Enlace, SEE es Si Existe Enlace.

$C=\left\{\begin{array}{cc}c_{i j k} / c_{i j k} & c_{i j k}=G \quad N E E \wedge c_{i j k}=c_{0} S E E \\ \forall i \in[0, N-1] \wedge j \in[0, N-1] \wedge k \in\left[0, n_{W}-1\right]\end{array}\right\}$

Donde $: N$ es el número de nodos de la red, $c_{0}$ es número de canales asociado a el tipo de red óptica que se está simulando y $n_{W}$ es número de longitudes de onda en la red óptica.

$\lambda=\left\{\begin{array}{cc}\lambda_{i j k} / \lambda_{i j k} & \lambda_{i j k}=0 W U \wedge \lambda_{i j k}=k \in Z^{+} W D \\ \forall i \in[0, N-1] & \wedge j \in[0, N-1] \wedge k \in\left[0, n_{W}-1\right]\end{array}\right\}$

Donde : $k$ es un número entero positivo asociado a cada longitud de onda, $W U$ es Longitud de Onda en Uso, $W D$ es Longitud de Onda en deshuso.

$T=\left\{\begin{array}{ccc}t_{i j k} / t_{i j k} & t_{i j k}=0 & N E E \wedge t_{i j k}=-t_{C} \in Z^{+} W U \wedge t_{i j k}=1 W D \\ \forall i \in[0, N-1] & \wedge j \in[0, N-1] \wedge k \in\left[0, n_{W}-1\right]\end{array}\right\}$

\section{ALGORITMO GENETICO (AGEN)}

El algoritmo genético se basa en la reproducción cromosómica al utilizar la combinación de los genes como elemento para encontrar los lightpaths. Sea $P_{0}$ la población inicial del algoritmo genético a utilizar, y $n_{P}$ el número de cromosomas que forman esta población inicial. Además, habrá $m$ algoritmos genéticos para los $m(m \leq N)$ nodos que solicitan servicio de transporte (Barpanda et al., 2011; Brandao et al., 2016; Kharroubi et al., 2015, Zakouni et al., 2017). Dentro de los algoritmos genéticos existen diversas implementaciones que permiten diferentes comportamientos de $n_{P}$ (políticas de regeneración o natalidad), para esta investigación este valor se mantiene constante y es un parámetro de simulación. De tal forma, se define la matriz tridimensional $P_{0}$ de $n_{P}\left(N+2 n_{w}+1\right) m$ elementos.

$P_{0}=\left\{\begin{array}{cc}p_{i j k} / p_{i j k} & p_{i 0 k}=n O \wedge p_{i(N-1) k}=n D \wedge p_{i j k}=\operatorname{Rand}(N) \\ \forall i \in\left[0, n_{P}-1\right] & \wedge j \in[1, N-2] \wedge k \in[0, m-1]\end{array}\right\}$

Donde : $n_{O}$ y $n_{D}$ son los nodo origen y destino del m-ésimo nodo solicitante, Rand $(N)$ es un número aleatorio entre 0 y $\mathrm{N}$-1. Esta matriz es llenada de manera aleatoria, las columnas adicionales son para guardar los cálculos de la función aptitud referente a cada longitud de onda disponible.

\section{ALGORITMO FLAGELOS BACTERIANOS (AFBA)}

Los microorganismos (MOO) son seres vivos entre lo que se encuentran los virus, las bacterias, hongos, levaduras y mohos, la estructura biológica es increíble. Las bacterias son MOO que en general poseen un elemento motriz denominado Flagelo, y se clasifican de acuerdo en Monótricas (un flagelo), Lofítrico (flagelos al lado), Anfítrico (flagelos polares) y Perítrico (flagelos perimetrales, ver figura 2) (Belas, 2014; Bonnie et al., 2015). Los flagelos son estructuras filamentosas que permiten el movimiento de las bacterias y en general son de tipo ondulado e inclusive helicoidales y mediante rotaciones logra el movimiento en cierta dirección. La heurística utilizada se basa en la ondulación y rotación de los flagelos, donde se aumentan los flagelos de monótricos hasta los lofítricos, para mejorar la potencia de la búsqueda (Rodriguez et al, 2019). En la figura 3 se muestra la matriz poblacional del nodo 6 de una topología de 14 nodos, para resolver la solicitud de conexión, los elementos $p_{i 06}$ y $p_{i(13) 6}$ de la matriz poblacional bacterial. 


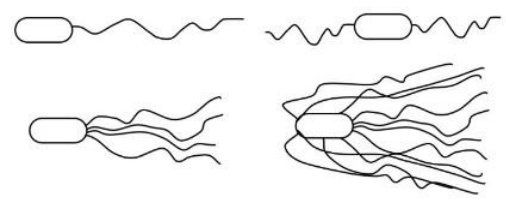

Fig. 2: Flagelos bacterianos monótricos y polítricos.
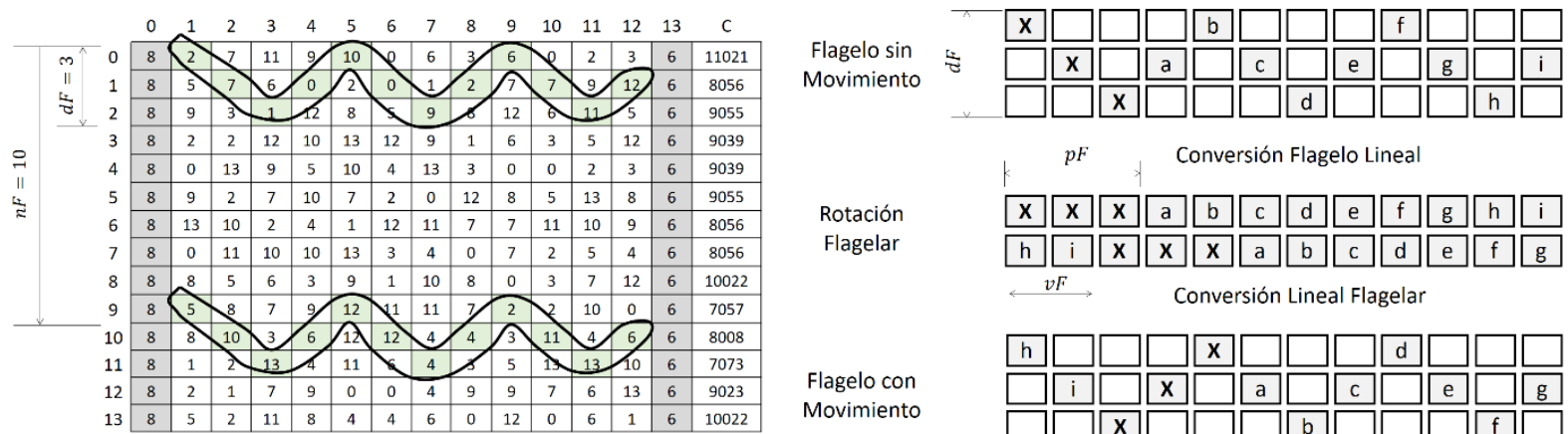

$p F$

Conversión Flagelo Lineal

Fig. 3: Matriz Bacterial para el ejemplo de 14 nodos.

Se observa la formación del flagelo en la matriz definiéndose cuatro variables; $n F$, indica el número de flagelos bacteriales $d F$, indica el diámetro de los flagelos; $v F$, indica la velocidad de rotación de los flagelos bacteriales y $p F$, indica la potencia de rotación de los flagelos. Además, se puede observar el proceso de rotación de los flagelos, donde las cuatro variables mencionadas son sujeto de optimización previa a la simulación. Luego de cada rotación se procede al recalculo de los costos por filas, ordenándose las filas en orden ascendente respecto de la columna de costos, luego se procede a eliminar una cantidad de filas de costos altos y son rellenadas nuevamente con valores aleatorios, las filas de la matriz poblacional bacterial no cambian siguiendo una política de crecimiento constante. A continuación, se recalculan los costos y se ordena ascendentemente y se repite el ciclo hasta encontrar el lightpath.

\section{ALGORITMO HIFAS FUNGICAS (AHFU)}

Los hongos poseen hifas que permiten el desarrollo replicador y garantizan la permanencia del microorganismo (Money, 2008). En la figura 4, se observan las hifas fúngicas y las células replicadas que garantizan el crecimiento continuo del MOO, la dirección de crecimiento está basada en la búsqueda de nutrientes (Figura 8) (Turra et al., 2016). Están formados por una columna de células alargadas y tubulares, envueltas por una pared celular, un grupo de hifas es denominado micelio. Las segmentaciones de las hifas presentan la división del filamento en celdas o también llamadas septos, donde la separación entre septos viene dada por tabiques, esto además de posicionar la célula en el septo permite la separación citoplasmática, por otro lado, no todos los hongos presentan esta morfología y en este caso se denominan cenocíticos (Rodriguez et al., 2018).

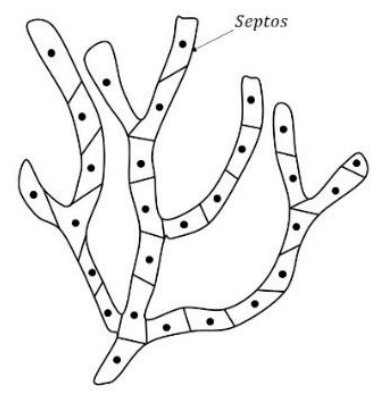

Fig. 4: Hifas de hongo mostrando sus septos.

Estos filamentos van creciendo en una dirección determinada orientados por la existencia de nutrientes en el entorno cercano, de esta forma puede garantizar su alimentación y desarrollo. Para el caso de la matriz poblacional cada nodo representa una espora (Figura 5), y dicho valor se replicará siguiendo la dirección de mayor costo o simplemente de existencia de costo dado que no se busca optimizar, solo se busca encontrar una ruta. Para el caso del algoritmo propuesto se utilizó el mejor costo, sin embargo, la complejidad temporal disminuiría si solo se utiliza un costo existente menor al número de canales máximo y diferente de 0 . Al iniciar las esporas en las filas de menor costos se transmiten propiedades positivas a las filas inferiores mejorando sus características al combinarlas con elementos de las filas con mejores costos. 


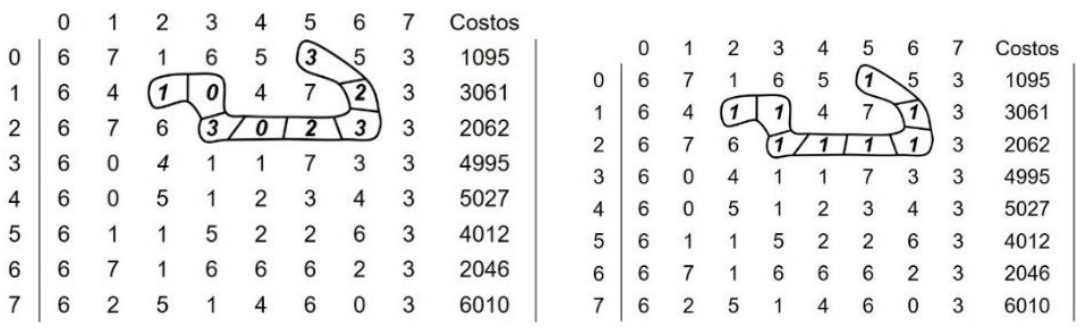

Fig. 5: Generación de la hifa para una espora.

\section{ESCENARIO DE SIMULACIÓN}

Realizada la simulación se compararon tres algoritmos similares bajo condiciones de simulación iguales AGEN, AFBA y AHFU. La red utilizada en la simulación fue la NSFNET (National Science Foundation Network) que posee 16 nodos y 25 enlaces de fibra óptica y 8 longitudes de onda (Ver Figura 6, se realizaron comparaciones de probabilidad de bloqueo y utilización de la red, variando la carga en el intervalo $[0,180]$ con incrementos de 10 Erlangs. El número de conexiones realizadas durante la simulación en los tres escenarios fue de $10^{8}$ solicitudes de conexión.

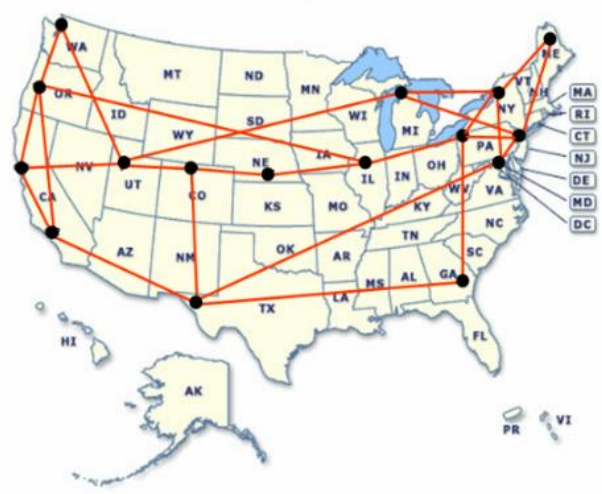

Fig. 6: Red NSFNET escenario de la simulación.

\section{RESULTADOS}

En la figura 7, se puede observar el comportamiento del indicador probabilidad de bloqueo $P_{B}$ de las microbioheurísticas (MBH) donde se observa que AGEN tiene mayor probabilidad de bloqueo que sus pares AFBA y AHFU, por lo que las dos últimas heurísticas se comportan con mejor rendimiento bajo todo el espectro de carga.
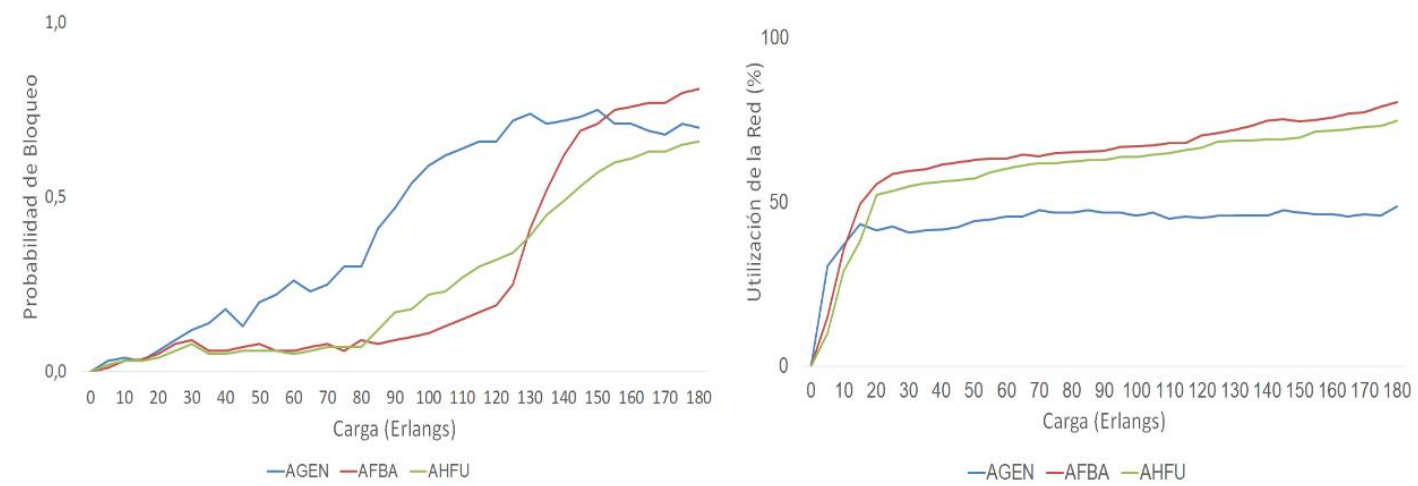

Fig. 7: Probabilidad de bloqueo.

Mientras que AFBA es mejor que AHFU hasta los a130 erlangs aproximadamente, mientras cuando se supera este límite (stress) AFBA desmejora y AHFU logra menor $P_{B}$. Esto permite inducir que los algoritmos AFBA y AHFU tienen el comportamiento de menor $P_{B}$, lo que es importante para los servicios demandados a la red de transporte óptico. Además, se puede observar el indicador utilización de la red $U_{R}$ que mide el consumo de los recursos, es decir el uso de los canales y enlaces en toda la red. Las MBH consumen de diferente forma los recursos, observándose que AGEN tiene menor $U_{R}$ que AFBA y AHFU, el cambio ocurre antes los 
10 egs por lo que tiene un mejor desempeño en este indicador. AFBA y AHFU tienen un uso de los recursos muy parecido siendo el menor AHFU, pero su diferencia no es significativa.
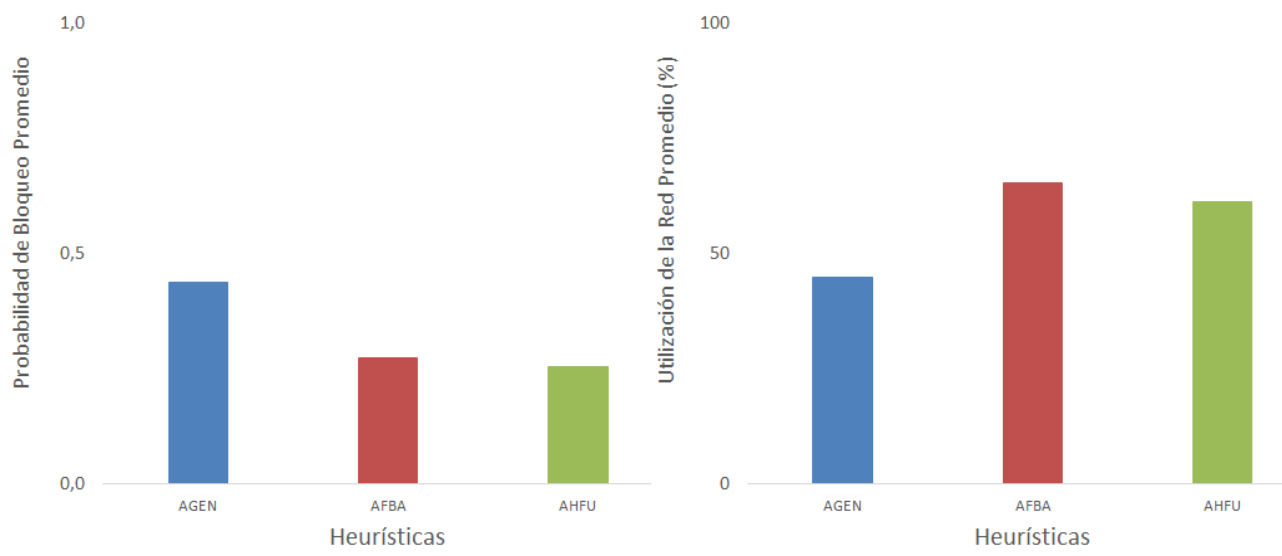

Fig. 8: Valor medio de la probabilidad de bloqueo.

En la figura 8, muestra el comportamiento heurístico, mediante el valor medio de $P_{B}$ en el dominio de la carga, y se puede observar que AHFU tiene el mejor comportamiento, esto refuerza que este algoritmo garantiza una alta atención al servicio solicitado a la red y muestra el comportamiento heurístico del valor medio de $U_{R}$, observándose que AGEN un significativo comportamiento de AGEN frente a sus pares, indicando que este algoritmo garantiza una alta disponibilidad para el futuro servicio solicitado a la red.

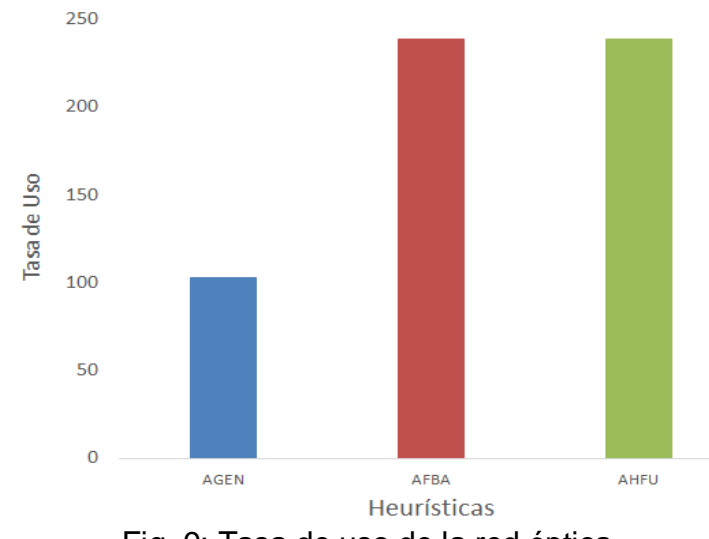

$T_{U}=\frac{U_{R}}{P_{B}}$

Dado que al analizar los dos indicadores $P_{B}$ y $U_{R}$ no se logra distinguir cual o cuales son los de mejor rendimiento en general se debe desarrollar un nuevo indicador que involucre ambos indicadores. La ecuación 7 , muestra el nuevo indicador denominado tasa de uso $\left(T_{U}\right)$, que nos indica cuanto de la utilización de la red es dado por cada punto de la probabilidad de bloqueo. La figura 9, muestra el comportamiento de la tasa de uso y es AGEN el que tiene mejor rendimiento en general.

\section{CONCLUSIONES}

El indicador $P_{B}$ permite evaluar la capacidad de los algoritmos de atender solicitudes entrantes a la red óptica, el $U_{R}$ evalúa la capacidad de atención de las solicitudes futuras y $T_{U}$ permite la comparación evaluando cuanto de la utilización de red se debe a la probabilidad de bloqueo. Si la red óptica requiere atender las solicitudes deberán basarse en el indicador $P_{B}$ y el AGEN no logra comportarse mejor que sus pares, sin embargo, si la red óptica requiere una mayor cobertura en los servicios futuros deberá utilizar el indicador $U_{R}$ y AFBA y AHFU no logran ser mejores que AGEN. Desde la perspectiva de evaluar los algoritmos heurísticos se utilizó $T_{U}$ que permite obtener una visión general de la algoritmia y resulta que AGEN tiene el mejor comportamiento promedio. Debe entenderse que esto no significa que sea mejor en todos los escenarios, queda claramente establecido que dependerá de los intereses instantáneos de la oferta de la red óptica. El Grupo de Investigación en Nuevas Tecnologías, tiene la seguridad que estos algoritmos pueden ser utilizados en otros procesos del ámbito industrial. 


\section{AGRADECIEMIENTOS}

Especial agradecimiento al Proyecto Dicyt: Código 081872RG, Vicerrectoría de Investigación, Desarrollo e Innovación de la Universidad de Santiago de Chile - USACH, al Proyecto IMP-ING-2660 de la Vicerrectoría de Investigaciones de la Universidad Militar Nueva Granada y a la Facultad Tecnológica de la Universidad de Santiago de Chile.

\section{REFERENCIAS}

Rosario, K., Ferreira dos Santos, A. y Ferreira, W., Hybrid Algorithms for Routing and Assignment Wavelengths in Optical Networks, Latin America Transactions, 8(3), 214- 220 (2010).

Balasis, F., Wang, X., Xu S. y Tanaka, Y., A dynamic physical impairment-aware routing and wavelength assignment scheme for 10/40/100 Gbps mixed line rate wavelength switched optical networks, Advanced Communication Technology, 2013 15th International Conference on Advanced Communications Technology (ICACT), PyeongChang, 116-121 (2013).

Sankar, R., Kumar, A., Sahoo, B. y Majhi, B., Genetic Algorithm techniques to solve Routing and Wavelength Assignment problem in Wavelength Division Multiplexing all-optical networks. Communication Systems and Networks (COMSNETS), Bangalore, 1-8 (2011).

Belas, R., Biofilms, flagella, and mechanosensing or surfaces by bacteria, Trends in Microbiology, 22(9), 517-527(2014).

Brandão, J.S., Noronha, T.F. y Ribeiro, C.C., A biased random-key genetic algorithm to maximize the number of accepted lightpaths in WDM optical networks, https://doi.org/10.1007/s10898-015-0389-x, J. Glob. Optim., 65, 813-835 (2016).

Jaumard, B. y Daryalal, M., Efficient Spectrum Utilization in Large Scale RWA Problems, in IEEE/ACM Transactions on Networking, 25(2), 1263-1278, (2017).

Chaban, B., Hughes, H.V. y Beeby, M., The flagellum in bacterial pathogens: For motility and a whole lot more, Seminars in Cell and Developmental Biology, 46, 91-103 (2015).

Duhamel, C., Mahey, P., Martins, A.X., Saldanha, R.R. y de Souza, M.C., Model-hierarchical column generation and heuristic for the routing and wavelength assignment problem, https://doi.org/10.1007/s10288-016-0309-z, 4OR-Q J. Oper. Res., 14, 201-220 (2016).

Kharroubi, F., He, J., Tang, J., Chen, M. y Chen, L., Evaluation performance of genetic algorithm and tabu search algorithm for solving the Max-RWA problem in all-optical networks, Journal Of Combinatorial Optimization, 30(4), 1042-1061(2015) Koganti, R. y Sidhu, D., Analysis of routing and wavelength assignment in large WDM networks, Procedia Computer Science 34, 71-78 (2014).

Montes-Castañeda, B., Patiño-Garzón, J., Puerto-Leguizamón, G., A comparative study of multiobjective computational intelligence algorithms to find the solution to the RWA problem in WDM networks, DYNA, 82(194), 221-229 (2015).

Piedrahita, E., Hernández, C., Pedraza, L. y Salcedo, O., Evaluación De Protocolos De Señalización En Multiprotocolo Generalizado De Conmutación De Etiquetas (GMPLS) Capaces De Conmutar Paquetes Y Longitudes De Ondas. Inf. Tecnol., 25(1), 55-66. (2014).

Rodriguez, A. y Ramirez, L. y Basile, F., Metaheuristic solution for the routing and wavelength assignment (RWA) problem in wavelength-division multiplexing (WDM) optical networks, Inf. Tecnol., 28(6), 133-146 (2017).

Rodriguez, A., Chávez, R. y Ganga, A., Routing Wavelength Assignment: Novel Heuristic Based on Bacterial Flagella for Optical Networks, ICEECE Conference, Penang, Malaysia (2019).

Rodríguez, A., Ramirez, J. y Martinez, J., Heuristic algorithms: Novel solution for RWA problem in WDM optical networks, Journal of Telecommunication, Electronic and Computer Engineering,10(1-4), 1-7 (2018).

Russo, N.A., Noriega, S.B. y Duchowicz, R., Implementación de Sistema Óptico para Grabado de Redes de Bragg en Fibra Óptica, Inf. Tecnol., 22(2) 121-130 (2011).

Simón, L., y Monzón, J.M., Identificación y Ajuste Paramétrico De Una Máquina Trifásica De Inducción Magnética usando Algoritmos Genéticos, Inf. Tecnol., 21(3) 105-114 (2010).

Xiaoweng, C., Li, B. y Zhang, Z., A Dynamic RWA Algorithm in a Wavelength-Routed All-Optical Network with Wavelength Converters, https://doi.org/10.1109/INFCOM.2003.1209202, IEEE INFOCOM 2003, 1795-1804 (2003).

Zakouni, A., Luo, J. y Kharroubi, F., Genetic algorithm and tabu search algorithm for solving the static manycast RWA problem in optical networks, Journal of Combinatorial Optimization, 33(2), 726-741 (2017).

Zhou, F., Ju, M. y Ait-Ouahmed, A., Joint Optimization for Multicast Provisioning in Mixed-Line-Rate Optical Networks With a Column Generation Approach, Journal Of Lightwave Technology, 36(3), 637-649(2018). 